\title{
Spot Speed versus Speed Limit on Malaysian East-Coast Expressway
}

\author{
${ }^{1} S$. Arintono \\ ${ }^{1}$ College of Engineering, Al Imam Mohammad ibn Saud Islamic University, Riyadh, Saudi Arabia \\ Email: SPArintono@imamu.edu.sa
}

\begin{abstract}
Speed is one of the important causal factors for accidents on the road. This study aims to investigate the effectiveness of the speed warning sign on Malaysia's East Coast Expressway where the rate of accident is high. Two points were selected, one representing locations far from any speed related warning sign, and one location immediately after a speed warning sign is in sight, to assess the significance of the differences of the spot speeds. Results of the analysis suggest that the majority of vehicles $(63.24 \%)$ travel over the speed limit and that the speed warning sign does function in reducing the mean spot speed. However, the speed reduction is not to the desired level, i.e., under the speed limit. Other strategies have to be introduced to achieve the target.
\end{abstract}

Keywords spot speed, speed limit, warning sign, expressway Paper type Research paper

\section{INTRODUCTION}

The maximum speed limits on Malaysian Expressways range from 90 kilometers per hour (kph) on segments within urban and suburban areas to $110 \mathrm{kph}$ on rural segments. For example, on the East-Coast Expressways (ECE or E8) the maximum speed limit is $90 \mathrm{kph}$ on the section between Gombak and Karak, and is increased to $110 \mathrm{kph}$ on the Karak-Jabor (Phase 1) and Jabor-Kuala Terengganu (Phase 2) sections.

It has been widely accepted that speed is one of the important causal factors which affect accidents and its severity. Therefore, usually there are traffic signs indicating maximum speed limit, warning signs, and speed detection systems along expressways as a reminder to the motorists to comply with the regulations. The speed detection system may consist of a speed camera as part of the Automatic Enforcement System (AES), which automatically takes a photograph of the speeding vehicles. Based on this evidence the owner of the vehicles will be notified to pay the fine. The rate of the fine starts from MYR150.00 at the initial stage, which increases to MYR200.00 after two weeks if it is not paid, and finally to MYR300.00. The AES speed cameras are mainly installed at the North-South Expressways (E1 and E2) and some of the urban highways within the Federal Territory of Kuala Lumpur and Putrajaya.

In addition to the AES camera, additional manual speed detection system is also applied on the expressways, ECE being a prime example. There, on a routine basis, traffic police monitor the spot speed of the passing vehicles. Those who are over the speed limit (90 or $110 \mathrm{kph}$ depending on the location) will be photographs and fined. However, probably due to the problem on the availability of staff and equipment, this speed monitoring cannot be conducted following a fixed or continuous schedule, leaving some 'blank spots' in terms of location and time, enabling motorist to speed up 'safely' (undetected). It is also important to note that, so far, the speed monitoring is only conducted during the day (roughly within working hours of 9:00-17:00), meaning that road users have 'more freedom' to drive at high speed during the night. The present study embarks on a hypothesis that the presence of a speed warning sign serves as a strong message to motorists to adjust their speed to comply with the speed limit regulation.

\section{SPeEd Limit Versus Driving Speed}

It is a common practice that people usually drive over the posted speed limit, especially when there is no visible enforcement system in place. Therefore various methods have been put in trial in order to make people obey the speed limit regulation which is related to road safety. The following paragraphs discuss the importance for the authorities to maintain the speed limit, and for the road users to drive within the posted speed limit.

There have been some proposals to raise the speed limit from 55 to $65 \mathrm{mph}$ on the state-owned, rural, nonfreeway highway in the United States. The associated benefits and dis-benefits were evaluated, which include reduced travel time, increased fuel consumption, and increased traffic crashes. The benefit from travel time saving exceeds the fuel consumption costs. However, the total benefit was far outweighed by crash related dis-benefits and agency costs for the infrastructure modification, signaling that raise in speed limit should be implemented with care [1]. The facts that in 
Malaysian highways and expressways the speed limits are either $90 \mathrm{kph}(55 \mathrm{mph})$ or $110 \mathrm{kph}(65 \mathrm{mph})$ shows they are perfectly within the ideal limits.

Spot Speed versus Speed Limit on Malaysian East-Coast Expressway.

In Norway, a cost-benefit analysis was conducted on the rewarding system for not speeding, in which drivers receive incentives amounting up to USD1586 per year (at 2013 prices) depending on the level of the relative accident rate. The monetary valuations of the prevention of traffic fatalities and injuries were applied in the scheme. However, it turns out that the benefits were found to be smaller than the costs involved, that the scheme would not sustain if implemented [2].

Considerable samples of spot speed from Riyadh and Buraydah in Saudi Arabia reveal that drivers tend to drive significantly over the speed limit. The proportions of motorists with 85th percentile speeds over the speed limit are $10 \%$, $88 \%$, and $100 \%$ on freeways, arterials, and residential streets, respectively. The primary concern is the relatively high speed driving habit through residential streets where pedestrian activities are prevalent. The local study also shows that enforcement methods, e.g., the presence of speed camera or police car, appeared to be effective in reducing the mean speed by at least $10 \mathrm{kph}[3]$.

An analysis was conducted on the effect of change of speed limit on the driving speed on interurban roads. The ratio between mean driving speed before to mean driving speed after the speed limit change was evaluated. Result of the study shows that the mean driving speed increases or decreases significantly, nearly proportional to the increase or decrease of the posted speed limit [4]. Another study by Mekker et al. [5] states that drivers need to observe multiple signs in a variable speed limit road environment before any reduction in speed finally occur. Example from the study shows that a $15 \mathrm{mph}$ sign of speed limit drop will result in $7.5 \mathrm{mph}$ (median speed) reduction in driving speed and that only $4 \%$ of cars and $10 \%$ of trucks comply the new speed limit. This finding highlights the importance of providing repeated signs in areas with the variable speed limit, and that the actual speed reduction is less than what is intended.

\section{METHOD}

The current study was conducted along ECE Phase 1 and 2, where ECE Phase 1 is fitted with speed warning signs, whereas such warning signs are rarely encountered on ECE Phase 2. Two points were selected along ECE Phase 1, the first one represents a location far from any speed warning sign where motorist are assumed to drive at high speed, and a location soon after the warning sign is in sight in which case drivers should have slowed down their vehicle speed. On ECE Phase 2 on the other hand, only one point is selected, at a long straight stretch of the expressway where road users are assumed to maximize speed.

One of the many speed warning signs (which read 'Camera Operation Zone') within ECE Phase 1 is situated at

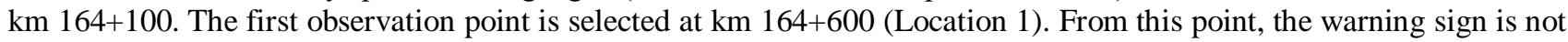
in sight yet, which means that when passing the particular point vehicles still run at high speed. The second observation point is located at $\mathrm{km} 163+600$ (Location 2), half a kilometer away from (after) the warning sign in the direction towards Kuala Lumpur where drivers are expected to have reduced the speed of their vehicles to comply with the $110 \mathrm{kph}$ speed limit. Another observation point is located at $\mathrm{km} 273+000$ (Location 3) on a long straight section of ECE Phase 2 where vehicles are expected to move at their maximum desirable speed.

Various types of vehicle use the expressway ranging from the passenger car, commercial vehicles (bus and truck), and motorcycle. The focus of the study is on passenger car which also includes multi-purpose-vehicle (MPV) and sport-utility-vehicle (SUV), since they have similar operating characteristics in terms of speed. However, samples were also taken for bus, truck, and motorcycle.

The presence of an observer (surveyor) wearing reflecting color safety jacket and handling a speed gun while escorted by traffic police by the road side or in the central median is believed to have some impacts to the road users in terms of their selected driving speed. However, it is an unavoidable condition. The best which can be done is for the observer to hide behind a bridge abutment or tree.

Spot speed observation was conducted twice at each location on a typical weekday and a weekend in order to reveal any difference between the days since during weekend many of the travelers travel with family which may affect the selection of the travel speed. Duration of the observation was set at 1-2 hours until sufficient numbers of the vehicle were observed at each location.

\section{DISCUSSION}

A series of observation was conducted on weekdays of 25 Oct (Tue), 26 Oct (Wed) and weekends of 29 Oct (Sat) and 30 Oct (Sun) of the Year 2016 on ECE Phase 1 (two locations) and ECE Phase 2 (one location). Summary of the results in terms of the distribution of the sample size is shown in Table 1. The total number of vehicles observed within one-hour period depends on the intensity of the traffic and is roughly about $60 \%$ of the total number of the passing vehicles. 


\section{Spot Speed versus Speed Limit on Malaysian East-Coast Expressway}

TABLE I. Distribution of Spot SpeEd SAMPLE

\begin{tabular}{cccccc}
\hline Location & Date & Day & Period & Observed & Total vehicles \\
\hline 1 & $26-$ Oct & weekday & 10:00-11:00 & 88 & 125 \\
1 & $30-$ Oct & weekend & 10:00-11:00 & 103 & 172 \\
2 & $26-$-Oct & weekday & $16: 00-17: 00$ & 60 & 138 \\
2 & $30-$-Oct & weekend & $16: 00-17: 00$ & 116 & 218 \\
3 & $25-$-Oct & weekday & $10: 00-11: 00$ & 77 & 109 \\
3 & $29-$-Oct & weekend & $10: 00-11: 00$ & 87 & 139 \\
\hline
\end{tabular}

The majority of vehicles in the passenger car group, which include multi-purpose vehicle (MPV) and sport utility vehicle (SUV) and also motorcycle traveled at above the speed limit of $110 \mathrm{kph}$ as shown in Table 2, whereas the bus and truck in the commercial vehicle group below the speed limit. Further discussion will be focused on passenger car group, trying to assess whether the presence of speed warning sign is effective to convey the message to drivers to comply with the speed limit regulation.

TABLE II. Average SpOt SPEED (KPH)

\begin{tabular}{cccccccc}
\hline Location & Day & Car & MPV & SUV & Motor & Truck & Bus \\
\hline 1 & weekday & 127.15 & 120.78 & 131.73 & 111.27 & 85.90 & 100.43 \\
1 & weekend & 113.50 & 110.00 & 107.11 & 103.13 & 79.85 & 89.50 \\
2 & weekday & 112.69 & 104.10 & 111.71 & 120.33 & 75.80 & 82.00 \\
2 & weekend & 112.76 & 111.13 & 114.76 & 115.40 & 67.00 & 90.00 \\
3 & weekday & 127.88 & 121.37 & 122.14 & 110.67 & 90.78 & 90.50 \\
3 & weekend & 122.91 & 110.07 & 119.40 & n/a & 89.86 & 96.50 \\
\hline \multirow{2}{*}{ Average } & & 119.48 & 112.91 & 117.81 & 112.16 & 85.36 & 87.65 \\
\hline
\end{tabular}

Table 3 shows the percentage of the vehicle which travels over the speed limit in the passenger car group. As expected, fewer vehicles travel above the speed limit on the weekend compared to weekday except in Location 2 where more vehicles travel over the speed limit on the weekend rather than a weekday. However, the difference is insignificant (50.93\% vs 51.24\%). One of the reasons could be that for weekend trips people go with the family, and so they have to be more careful in driving, evidenced by the lower observed spot speed.

TABLE III. Percent Over The SPEed Limit

\begin{tabular}{ccccc}
\hline Location & Day & Sample & Over & \% over \\
\hline 1 & weekday & 92 & 75 & 81.52 \\
1 & weekend & 143 & 89 & 62.24 \\
2 & weekday & 108 & 55 & 50.93 \\
2 & weekend & 201 & 103 & 51.24 \\
3 & weekday & 90 & 70 & 77.78 \\
3 & weekend & 114 & 81 & 71.05 \\
\hline Total & & 748 & 473 & 63.24 \\
\hline
\end{tabular}

Summary of the main findings is presented in Table 4. As described earlier, Locations 1 and 3 are away from any speed warning sign where vehicles may travel faster than at Location 2 which is situated just after the speed warning sign is in sight.

Comparing the means of the spot speed during the weekday, between Location 1 (126.09 kph) and Location 2 $(110.38 \mathrm{kph})$ the difference is significant, meaning that the presence of warning sign is effective in reducing the speed limit. However, the reduction is not to the desired level. Instead of being reduced to under the speed limit of $110 \mathrm{kph}$, the resulting speed is still right at the borderline $(110.38 \mathrm{kph})$. 
TABLE IV.

Distribution OF SPOT SPEED By LOCATION

\begin{tabular}{ccccccc}
\hline \multirow{2}{*}{ Statistics } & Location 1 & \multicolumn{3}{c}{ Location 2 } & \multicolumn{3}{c}{ Location 3 } \\
\cline { 2 - 6 } & Weekday & Weekend & Weekday & Weekend & Weekday & Weekend \\
\hline Mean & 126.09 & 112.06 & 110.38 & 112.55 & 125.61 & 120.67 \\
StDev & 15.53 & 23.05 & 18.92 & 19.14 & 17.99 & 18.82 \\
Max & 172 & 167 & 149 & 167 & 176 & 172 \\
Min & 100 & 60 & 67 & 64 & 86 & 74 \\
Sample & 92 & 143 & 108 & 201 & 90 & 114 \\
\hline
\end{tabular}

Comparison of the means between Location 1 (126.09 $\mathrm{kph})$ and Location 3 (125.61 kph) shows that the difference is not significant. This tells us that in the absence of speed warning sign road users tend to drive at their maximum desirable speed which is comparable irrespective of the expressway section they are driving in, either with or without speed warning signs.

Comparison of the mean spot speed during the weekend shows no significant difference between Location 1 $(112.06 \mathrm{kph})$ and Location $2(112.55 \mathrm{kph})$. However, the difference becomes significant if they are compared to Location $3(120.67 \mathrm{kph})$. The following explanation may apply.

In general, ECE Phase 2 is lightly trafficked compared to ECE Phase 1, which is more or less reflected by the volume of vehicles in the right most column of Table 1 (locations 1 and 2 are located in ECE Phase 1, location 3 is located in ECE Phase 2). The traffic volume on that particular observation day could be in such level that the maximum desirable speed was maintained at around $112 \mathrm{kph}$, close enough to the speed limit of $110 \mathrm{kph}$, that drivers do not feel any necessity to adjust or slow down. On the other hand, being in ECE Phase 2 where most drivers believe that there is no speed warning sign, coupled with the light traffic condition, the resulting speed is higher at $120.67 \mathrm{kph}$ at Location 3.

Similar comparison between weekday $(126.09 \mathrm{kph})$ and weekend $(112.06 \mathrm{kph})$ for Location 1 results in a significant difference at 0.05 level. The reason behind the lower speed during the weekend compared to a weekday could be that during weekday many of the intercity travelings involves business activity which is constrained by time (implying the need to travel faster), while traveling during weekend usually with family members is in a more relaxing environment, which implies lower speed. Similar trend and explanation apply for Location 3 (125.61 kph weekday vs $120.67 \mathrm{kph}$ weekend).

The average spot speed in Location 2 during the weekday $(110.38 \mathrm{kph})$ is comparable to the weekend (112.55 $\mathrm{kph}$ ), i.e., the difference is not significant. Again, it is believed that the presence of a warning sign plays its role in reducing the average speed of the passing vehicles. However, the message is not strong enough to enable the average speed drop to below the speed limit.

Some drawbacks remain in the methodology part which reduces the strength of the finding. One example is the positions of the observation points, Location 1 and 3. Location 1 at $\mathrm{km} 164+600$ is situated just $500 \mathrm{~m}$ before the speed camera warning sign. At this relatively short distance, the message contained in the warning sign is still unreadable, but the color (bright yellow) to some motorists may have been perceived as indicative of a speed camera warning sign, as this type of warning sign is scattered along the expressway, on average at 10-20 kilometer intervals. So, they may have started to slow down. In short, the peak of the sought-after spot speed is thus undetected.

Location 3 at $\mathrm{km} \mathrm{273+000} \mathrm{is} \mathrm{situated} \mathrm{at} \mathrm{the} \mathrm{Cheneh} \mathrm{Junction} \mathrm{(Exit} \mathrm{839)} \mathrm{observing} \mathrm{traffic} \mathrm{in} \mathrm{the} \mathrm{direction}$ towards Kuala Lumpur. Since it is close to a junction some of the passing traffic, those who just enter the expressway, or those intending to leave the expressway, are not at their maximum desirable speed. Also, there are speed limit signs nearby, which means that the traffic is not entirely free from 'persuasion' to stay under the speed limit. In other words, again, the highest peak of the spot speed in ECE Phase 2 is not detected.

Result of this research is comparable to that in Thailand, where motorcycle traffic is under speed enforcement camera in urban arterial streets of Khon Kaen city. There, the observed spot speed after is significantly reduced compared to before installation of the speed enforcement camera, i.e., the $85^{\text {th }}$ percentile, whereas the mean speed is relatively constant [6]. Another research conducted in Abu Dhabi, United Arab Emirates, also confirms that driver's behavior towards speed limit compliance is affected by the presence of speed management devices (speed camera, speed radar, speed limit and speed warning sign, enforcement police, etc.) [7]. It shows the importance of speed monitoring effort to improve safety on the road.

\section{CONCLUSION}

The speed limit at the main sections of Malaysia's East-Coast Expressway is $110 \mathrm{kph}$, and speed warning signs which read 'Camera Operation Zone' are installed along the expressway at 10-20 kilometer intervals. Results of the observation show that the majority of vehicles $(63.24 \%)$, especially in the passenger car group and motorcycle, maintain a driving 


\section{Spot Speed versus Speed Limit on Malaysian East-Coast Expressway}

speed above the limit of $110 \mathrm{kph}$, whereas commercial vehicles (bus and truck), because of the size and weight, stay under the speed limit.

The presence of the speed warning sign has been successful in reducing the spot speed. However, the magnitude of the speed reduction is not sufficient to draw the average speed to under the speed limit. Probably, it is necessary to install repetitive, consecutive warning signs at closer intervals (10 km at most rather than $20 \mathrm{~km})$ to convey a stronger message.

So far, the speed monitoring for enforcement is not conducted continuously on a 24/7 basis due to non-availability of staff and equipment, leaving some gaps in terms of space and time where motorists may travel at well over the speed limit which is highly risky for other road users as well. It is recommended that an automatic enforcement system (AES) is installed and activated shortly in order to reduce the number and fatality of accidents.

\section{ACKNOWLEDGMENT}

The author wishes to thank Universiti Malaysia Pahang (UMP) and Management of ANIH Berhad, the operator of ECE Phase 1 and 2, for the given opportunity to conduct the study, and special thanks to Wahyu Nur Susiana Omar, student of UMP who contributed in the data collection and analysis.

\section{REFERENCES}

[1] T.J. Gates, P.T. Savolainen, and J.J. Kay, "Economic Analysis of 65 mph Speed Limits on Rural Highways,” Transportation Research Record, Issue 2597, 2016, page 60-66.

[2] R. Elvik, "Cost-Benefit Analysis of Incentive Systems Rewarding Compliance with Speed Limits," Transportation Research Record, Issue 2465, 2014, page 8-15.

[3] A.S. Al-Ghamdi, "Analysis of Speeding in Saudi Arabia and Effectiveness of Enforcement Methods," Transportation Research Record, Issue 1969, 2006, page 1-9.

[4] O. Musicant, H. Bar-Gera and E. Schechtman, "Impact of Speed Limit Change on Driving Speed and Road Safety on Interurban Roads Meta Analysis," Transportation Research Record, Issue 2601, 2016, page 42-49.

[5] M.M. Mekker, S.M. Remias, W.A. Bunnell, D.W. Krohn, E.D. Cox, and D.M. Bullock, "Variable Speed Limit Study Upstream of an In diana Work Zone with Vehicle Matching," Transportation Research Record, Issue 2555, 2016, page 53 -64

[6] J. Kumphong, T. Satiennam, W. Satiennam, and S. Tirapat, "Change of Motorcycle Speed under Speed Enforcement Camera on Urban Arterial in Khon Kaen City, Thailand," International Journal of GEOMATE, Vol 16, Issue 56, April 209, pp 159-164.

[7] H. Hany, M. Shawky, and M.K. Mohamed, "Investigation of Driver's Behavior towards Speeds using Crash Data and Self-Reported Questionnaire," Accident Analysis and Prevention, Vol 98, pp 348-358, Jan 2017. 